\title{
Modified operational matrix method for second-order nonlinear ordinary differential equations with quadratic and cubic terms
}

\author{
Burcu Gürbüz ${ }^{a, b, c^{*}}$ and Mehmet Sezer ${ }^{d}$ \\ ${ }^{a}$ Department of Computer Engineering, Üsküdar University, Turkey \\ ${ }^{b}$ Institute of Mathematics, Johannes Gutenberg-University Mainz, Germany \\ ${ }^{c}$ Jean Leray Mathematics Laboratory, University of Nantes, France \\ ${ }^{d}$ Department of Mathematics Manisa Celal Bayar University, Turkey \\ burcu.gurbuz@uskudar.edu.tr, mehmet.sezer@cbu.edu.tr
}

\section{ARTICLE INFO}

Article History:

Received 30 May 2019

Accepted 16 December 2019

Available 01 July 2020

Keywords:

Nonlinear ordinary differential equations

Laguerre polynomials and series

Collocation points

Residual error estimation

AMS Classification 2010:

\section{ABSTRACT}

In this study, by means of the matrix relations between the Laguerre polynomials, and their derivatives, a novel matrix method based on collocation points is modified and developed for solving a class of second-order nonlinear ordinary differential equations having quadratic and cubic terms, via mixed conditions. The method reduces the solution of the nonlinear equation to the solution of a matrix equation corresponding to system of nonlinear algebraic equations with the unknown Laguerre coefficients. Also, some illustrative examples along with an error analysis based on residual function are included to demonstrate the validity and applicability of the proposed method.

\section{$(\mathrm{cc}) \mathrm{BY}$}

\section{Introduction}

Nonlinear differential equations and the related initial and boundary value problems play an important role in astrophysics, physics and engineering. In recent years, to solve these problems both analytically and numerically which have applications in various branches of pure and applied sciences, several numerical and analytical methods have been given. But it may not be possible to find the analytical solutions of such problems for all coefficient functions.

These type of mathematical models can be described by particular names such as Riccati equation, nonlinear equations of motion, Duffing's equation, Van Der Pol's equation, the equation of motion with quadratic damping, Emden's equation, Liouville's equation [1 5].

In this study, we consider the second-order nonlinear ordinary differential equations with quadratic and cubic terms:

$$
\begin{aligned}
& \sum_{k=0}^{2} P_{k}(x) y^{(k)}(x)+\sum_{p=0}^{2} \sum_{q=0}^{p} Q_{p q}(x) y^{(p)}(x) y^{(q)}(x) \\
+ & \sum_{p=0}^{2} \sum_{q=0}^{p} \sum_{r=0}^{q} Q_{p q r}(x) y^{(p)}(x) y^{(q)}(x) y^{(r)}(x) \\
= & g(x), \quad 0 \leq x \leq b<\infty
\end{aligned}
$$

with the mixed conditions

$$
\sum_{k=0}^{1}\left(a_{k j} y^{(k)}(0)+b_{k j} y^{(k)}(b)\right)=\lambda_{j}, \quad j=0,1,
$$

where $P_{k}(x), Q_{p q}(x), Q_{p q r}(x)$ and $g(x)$ are functions defined on the interval $0 \leq x \leq b<\infty$; $a_{k j}, b_{k j}$ and $\lambda_{j}$ are appropriate and real constants; $y(x)$ is an unknown function to be determined [6].

*Corresponding Author 
In this study, we develop a new numerical methods to find the approximate solutions of Eq. (1) in the truncated Laguerre series form

$$
y(x) \cong y_{N}(x)=\sum_{n=0}^{N} a_{n} L_{n}(x), \quad 0 \leq x \leq b<\infty,
$$

where $a_{n}, n=0,1, \ldots, N, N \geq 2$ are the unknown Laguerre coefficients and $L_{n}(x), n=0,1, \ldots, N$ are the Laguerre functions of first kind defined by

$$
L_{n}(x)=\sum_{k=0}^{n} \frac{(-1)^{k}}{k !}\left(\begin{array}{l}
n \\
k
\end{array}\right) x^{k}, \quad 0 \leq x \leq b<\infty .
$$

\section{Operational matrix relations}

Firstly, let us write Eq. (1) in the form

$$
L[y(x)]+N_{2}[y(x)]+N_{3}[y(x)]=g(x),
$$

where the linear ordinary differential part

$$
L[y(x)]=\sum_{k=0}^{2} P_{k}(x) y^{(k)}(x),
$$

the nonlinear quadratic part

$$
N_{2}[y(x)]=\sum_{p=0}^{2} \sum_{q=0}^{p} Q_{p q}(x) y^{(p)}(x) y^{(q)}(x),
$$

and the nonlinear cubic part

$N_{3}[y(x)]=\sum_{p=0}^{2} \sum_{q=0}^{p} \sum_{r=0}^{q} Q_{p q r}(x) y^{(p)}(x) y^{(q)}(x) y^{(r)}(x)$.

\subsection{Matrix representation of linear ordinary differential part}

Now, we consider Eq.(1) and find the matrix forms of each term in the equation. So, we convert Laguerre polynomial solution (3) to the matrix form as

$$
\begin{aligned}
y(x)=y^{(0)}(x) & \cong \mathbf{L}(x) \mathbf{A}, \\
y^{(1)}(x) & =\mathbf{L}(x) \mathbf{C A}, \\
y^{(2)}(x) & =\mathbf{L}(x) \mathbf{C}^{2} \mathbf{A},
\end{aligned}
$$

where

$$
\begin{aligned}
\mathbf{L}(x) & =\left[\begin{array}{cccc}
L_{0}(x) & L_{1}(x) & \cdots & L_{N}(x)
\end{array}\right] \\
\mathbf{C} & =\left[\begin{array}{ccccc}
0 & -1 & -1 & \cdots & -1 \\
0 & 0 & -1 & \cdots & -1 \\
\vdots & \vdots & \vdots & \ddots & \vdots \\
0 & 0 & 0 & \cdots & -1 \\
0 & 0 & 0 & \cdots & 0
\end{array}\right] \\
\mathbf{A} & =\left[\begin{array}{llll}
a_{0} & a_{1} & \cdots & a_{N}
\end{array}\right]^{T}
\end{aligned}
$$

\subsection{Matrix representation of nonlinear quadratic part}

Now, we consider matrix representation of nonlinear quadratic part. So, we define the matrices with related to (7) and (9)

$$
\begin{aligned}
\left(y^{(0)}(x)\right)^{2} & =\mathbf{L}(x) \overline{\mathbf{L}}(x) \overline{\mathbf{A}}, \\
y^{(1)}(x) y^{(0)}(x) & =\mathbf{L}(x) \mathbf{C} \overline{\mathbf{L}}(x) \overline{\mathbf{A}}, \\
\left(y^{(1)}(x)\right)^{2} & =\mathbf{L}(x) \mathbf{C} \overline{\mathbf{L}}(x) \overline{\mathbf{C A}}, \\
y^{(2)}(x) y^{(1)}(x) & =\mathbf{L}(x) \mathbf{C}^{2} \overline{\mathbf{L}}(x) \overline{\mathbf{C A}}, \\
y^{(2)}(x) y^{(0)}(x) & =\mathbf{L}(x) \mathbf{C}^{2} \overline{\mathbf{L}}(x) \overline{\mathbf{A}}, \\
\left(y^{(2)}(x)\right)^{2} & =\mathbf{L}(x) \mathbf{C}^{2} \overline{\mathbf{L}}(x) \overline{\mathbf{C}^{2}} \overline{\mathbf{A}},
\end{aligned}
$$

where

$$
\begin{aligned}
\overline{\mathbf{L}}(x) & =\operatorname{diag}\left[\begin{array}{llll}
\mathbf{L}(x) & \mathbf{L}(x) & \cdots & \mathbf{L}(x)
\end{array}\right], \\
\overline{\mathbf{C}} & =\operatorname{diag}\left[\begin{array}{llll}
\mathbf{C} & \mathbf{C} & \cdots & \mathbf{C}
\end{array}\right], \\
\overline{\mathbf{A}} & =\left[\begin{array}{llll}
a_{0} \mathbf{A} & a_{1} \mathbf{A} & \cdots & a_{N} \mathbf{A}
\end{array}\right]^{T} .
\end{aligned}
$$

\subsection{Matrix representation of nonlinear cubic part}

Let us consider (8) as

$$
\begin{aligned}
N_{3}[y(x)] & =\sum_{p=0}^{2} \sum_{q=0}^{p} \sum_{r=0}^{q} Q_{p q r}(x) y^{(p)}(x) y^{(q)}(x) y^{(r)}(x) \\
& +Q_{000}(x) y^{(0)}(x) y^{(0)}(x) y^{(0)}(x) \\
& +Q_{100}(x) y^{(1)}(x) y^{(0)}(x) y^{(0)}(x) \\
& +Q_{110}(x) y^{(1)}(x) y^{(1)}(x) y^{(0)}(x) \\
& +Q_{111}(x) y^{(1)}(x) y^{(1)}(x) y^{(1)}(x) \\
& +Q_{200}(x) y^{(2)}(x) y^{(0)}(x) y^{(0)}(x) \\
& +Q_{210}(x) y^{(2)}(x) y^{(1)}(x) y^{(0)}(x) \\
& +Q_{211}(x) y^{(2)}(x) y^{(1)}(x) y^{(1)}(x) \\
& +Q_{220}(x) y^{(2)}(x) y^{(2)}(x) y^{(0)}(x) \\
& +Q_{221}(x) y^{(2)}(x) y^{(2)}(x) y^{(1)}(x) \\
& +Q_{222}(x) y^{(2)}(x) y^{(2)}(x) y^{(2)}(x)
\end{aligned}
$$


So, we define the matrices as

$$
\begin{aligned}
\left(y^{(0)}(x)\right)^{3} & =\mathbf{L}(x) \overline{\mathbf{L}}(x) \overline{\overline{\mathbf{L}}}(x) \overline{\overline{\mathbf{A}}}, \\
y^{(1)}(x)\left(y^{(0)}(x)\right)^{2} & =\mathbf{L}(x) \mathbf{C} \overline{\mathbf{L}}(x) \overline{\overline{\mathbf{L}}}(x) \overline{\overline{\mathbf{A}}} \\
\left(y^{(1)}(x)\right)^{2} y^{(0)}(x) & =\mathbf{L}(x) \mathbf{C} \overline{\mathbf{L}}(x) \overline{\mathbf{C}}(x) \overline{\overline{\mathbf{A}}}, \\
\left(y^{(1)}(x)\right)^{3} & =\mathbf{L}(x) \mathbf{C} \overline{\mathbf{L}}(x) \overline{\overline{\mathbf{C}}}(x) \overline{\overline{\mathbf{C A}}}, \\
y^{(2)}(x)\left(y^{(0)}(x)\right)^{2} & =\mathbf{L}(x) \mathbf{C}^{2} \overline{\mathbf{L}}(x) \overline{\overline{\mathbf{L}}}(x) \overline{\overline{\mathbf{A}}}, \\
y^{(2)}(x) y^{(1)}(x) y^{(0)}(x) & =\mathbf{L}(x) \mathbf{C}^{2} \overline{\mathbf{L}}(x) \overline{\mathbf{C}} \overline{\overline{\mathbf{L}}}(x) \overline{\overline{\mathbf{A}}},
\end{aligned}
$$

$$
\begin{aligned}
y^{(2)}(x)\left(y^{(1)}(x)\right)^{2} & =\mathbf{L}(x) \mathbf{C}^{2} \overline{\mathbf{L}}(x) \overline{\mathbf{C}} \overline{\mathbf{L}}(x) \overline{\overline{\mathbf{C A}}}, \\
\left(y^{(2)}(x)\right)^{2} y^{(0)}(x) & =\mathbf{L}(x) \mathbf{C}^{2} \overline{\mathbf{L}}(x) \overline{\mathbf{C}^{2}} \overline{\overline{\mathbf{L}}}(x) \overline{\overline{\mathbf{A}}} \\
\left(y^{(2)}(x)\right)^{2} y^{(1)}(x) & =\mathbf{L}(x) \mathbf{C}^{2} \overline{\mathbf{L}}(x) \overline{\mathbf{C}^{2}} \overline{\overline{\mathbf{L}}}(x) \overline{\overline{\mathbf{C A}}} \\
\left(y^{(2)}(x)\right)^{3} & =\mathbf{L}(x) \mathbf{C}^{2} \overline{\mathbf{L}}(x) \overline{\mathbf{C}^{2}} \overline{\overline{\mathbf{L}}}(x) \overline{\overline{\mathbf{C}^{2}}} \overline{\mathbf{A}},
\end{aligned}
$$

where

$$
\begin{aligned}
\overline{\overline{\mathbf{L}}}(x) & =\operatorname{diag}\left[\begin{array}{llll}
\overline{\mathbf{L}}(x) & \overline{\mathbf{L}}(x) & \ldots & \overline{\mathbf{L}}(x)
\end{array}\right], \\
\overline{\overline{\mathbf{C}}} & =\operatorname{diag}\left[\begin{array}{llll}
\overline{\mathbf{C}} & \overline{\mathbf{C}} & \ldots & \overline{\mathbf{C}}
\end{array}\right], \\
\overline{\overline{\mathbf{A}}} & =\left[\begin{array}{llll}
a_{0} \overline{\mathbf{A}} & a_{1} \overline{\mathbf{A}} & \cdots & a_{N} \overline{\mathbf{A}}
\end{array}\right]^{T} .
\end{aligned}
$$

\section{Method of solution}

Now, we define the collocation points as

$x_{i}=\frac{b}{N} i, \quad i=0,1, N ; 0 \leq x_{0}<x_{1}<\ldots<x_{N}=b$.

We substitute the collocation points (12) into Eq. (1), we have the system of matrix equations for $i=0,1, \ldots, N$,

$$
\begin{aligned}
& \sum_{k=0}^{2} P_{k}\left(x_{i}\right) y^{(k)}\left(x_{i}\right)+\sum_{p=0}^{2} \sum_{q=0}^{p} Q_{p q}\left(x_{i}\right) y^{(p)}\left(x_{i}\right) y^{(q)}\left(x_{i}\right) \\
+ & \sum_{p=0}^{2} \sum_{q=0}^{p} \sum_{r=0}^{q} Q_{p q r}\left(x_{i}\right) y^{(p)}\left(x_{i}\right) y^{(q)}\left(x_{i}\right) y^{(r)}\left(x_{i}\right) \\
= & g\left(x_{i}\right), \quad 0 \leq x \leq b<\infty,
\end{aligned}
$$

or briefly,

$$
\begin{aligned}
\sum_{k=0}^{2} \mathbf{P}_{k} \mathbf{Y}^{(k)} & +\sum_{p=0}^{2} \sum_{q=0}^{p} \mathbf{Q}_{p q} \mathbf{Y}^{(p, q)} \\
& +\sum_{p=0}^{2} \sum_{q=0}^{p} \sum_{r=0}^{q} \mathbf{Q}_{p q r} \mathbf{Y}^{(p, q, r)} \\
& =\mathbf{G}, \quad 0 \leq x \leq b<\infty
\end{aligned}
$$

where

$$
\begin{aligned}
\mathbf{P}_{k} & =\operatorname{diag}\left[\begin{array}{llll}
P_{k}\left(x_{0}\right) & P_{k}\left(x_{1}\right) & \cdots & P_{k}\left(x_{N}\right)
\end{array}\right], \\
\mathbf{Q}_{p q} & =\operatorname{diag}\left[\begin{array}{llll}
Q_{p q}\left(x_{0}\right) & Q_{p q}\left(x_{1}\right) & \cdots & Q_{p q}\left(x_{N}\right)
\end{array}\right], \\
\mathbf{Q}_{p q r} & =\operatorname{diag}\left[\begin{array}{llll}
Q_{p q r}\left(x_{0}\right) & Q_{p q r}\left(x_{1}\right) & \cdots & Q_{p q r}\left(x_{N}\right)
\end{array}\right],
\end{aligned}
$$

and

$$
\begin{aligned}
\mathbf{Y}^{(k)}= & {\left[\begin{array}{c}
y^{(k)}\left(x_{0}\right) \\
y^{(k)}\left(x_{1}\right) \\
\vdots \\
y^{(k)}\left(x_{N}\right)
\end{array}\right], \mathbf{G}=\left[\begin{array}{c}
g\left(x_{0}\right) \\
g\left(x_{1}\right) \\
\vdots \\
g\left(x_{N}\right)
\end{array}\right], } \\
\mathbf{Y}^{(p, q, r)}= & {\left[\begin{array}{c}
y^{(p)}\left(x_{0}\right) y^{(q)}\left(x_{0}\right) y^{(r)}\left(x_{0}\right) \\
y^{(p)}\left(x_{1}\right) y^{(q)}\left(x_{1}\right) y^{(r)}\left(x_{1}\right) \\
\vdots \\
y^{(p)}\left(x_{N}\right) y^{(q)}\left(x_{N}\right) y^{(r)}\left(x_{N}\right)
\end{array}\right], } \\
\mathbf{Y}^{(p, q)}= & {\left[\begin{array}{c}
y^{(p)}\left(x_{0}\right) y^{(q)}\left(x_{0}\right) \\
y^{(p)}\left(x_{1}\right) y^{(q)}\left(x_{1}\right) \\
\vdots \\
y^{(p)}\left(x_{N}\right) y^{(q)}\left(x_{N}\right)
\end{array}\right] . }
\end{aligned}
$$

By the other hand, we can write following matrix forms of the nonlinear quadratic and nonlinear cubic parts from (8) and (9) for $p, q, r=0,1,2$

$$
\begin{aligned}
& \mathbf{Y}^{(0,0)}=\mathbf{L}_{(0,0)}^{*} \overline{\mathbf{A}}, \quad \mathbf{Y}^{(1,0)}=\mathbf{L}_{(1,0)}^{*} \overline{\mathbf{A}}, \\
& \mathbf{Y}^{(1,1)}=\mathbf{L}_{(1,1)}^{*} \overline{\mathbf{A}}, \quad \mathbf{Y}^{(2,0)}=\mathbf{L}_{(2,0)}^{*} \overline{\mathbf{A}}, \\
& \mathbf{Y}^{(2,1)}=\mathbf{L}_{(2,1)}^{*} \overline{\mathbf{A}}, \quad \mathbf{Y}^{(2,2)}=\mathbf{L}_{(2,2)}^{*} \overline{\mathbf{A}},
\end{aligned}
$$

and

$$
\begin{aligned}
& \mathbf{Y}^{(0,0,0)}=\mathbf{L}_{(0,0,0)}^{*} \overline{\overline{\mathbf{A}}}, \mathbf{Y}^{(1,0,0)}=\mathbf{L}_{(1,0,0)}^{*} \overline{\overline{\mathbf{A}}} \\
& \mathbf{Y}^{(1,1,0)}=\mathbf{L}_{(1,1,0)}^{*} \overline{\overline{\mathbf{A}}}, \mathbf{Y}^{(1,1,1)}=\mathbf{L}_{(1,1,1)}^{*} \overline{\overline{\mathbf{A}}}, \\
& \mathbf{Y}^{(2,0,0)}=\mathbf{L}_{(2,0,0)}^{*} \overline{\overline{\mathbf{A}}}, \mathbf{Y}^{(2,1,0)}=\mathbf{L}_{(2,1,0)}^{*} \overline{\overline{\mathbf{A}}}, \\
& \mathbf{Y}^{(2,1,1)}=\mathbf{L}_{(2,1,1)}^{*} \overline{\overline{\mathbf{A}}}, \mathbf{Y}^{(2,2,0)}=\mathbf{L}_{(2,2,0)}^{*} \overline{\overline{\mathbf{A}}}, \\
& \mathbf{Y}^{(2,2,1)}=\mathbf{L}_{(2,2,1)}^{*} \overline{\overline{\mathbf{A}}}, \mathbf{Y}^{(2,2,2)}=\mathbf{L}_{(2,2,2)}^{*} \overline{\overline{\mathbf{A}}},
\end{aligned}
$$

where 


$$
\begin{aligned}
& \mathbf{L}_{(0,0)}^{*}=\left[\begin{array}{c}
\mathbf{L}\left(x_{0}\right) \overline{\mathbf{L}}\left(x_{0}\right) \\
\mathbf{L}\left(x_{1}\right) \mathbf{\mathbf { L }}\left(x_{1}\right) \\
\vdots \\
\mathbf{L}\left(x_{N}\right) \overline{\mathbf{L}}\left(x_{N}\right)
\end{array}\right], \\
& \mathbf{L}_{(1,0)}^{*}=\left[\begin{array}{c}
\mathbf{L}\left(x_{0}\right) \mathbf{C} \overline{\mathbf{L}}\left(x_{0}\right) \\
\mathbf{L}\left(x_{1}\right) \mathbf{C} \overline{\mathbf{L}}\left(x_{1}\right) \\
\vdots \\
\mathbf{L}\left(x_{N}\right) \mathbf{C} \overline{\mathbf{L}}\left(x_{N}\right)
\end{array}\right] \text {, } \\
& \mathbf{L}_{(1,1)}^{*}=\left[\begin{array}{c}
\mathbf{L}\left(x_{0}\right) \mathbf{C} \overline{\mathbf{L}}\left(x_{0}\right) \overline{\mathbf{C}} \\
\mathbf{L}\left(x_{1}\right) \mathbf{C} \overline{\mathbf{L}}\left(x_{1}\right) \overline{\mathbf{C}} \\
\vdots \\
\mathbf{L}\left(x_{N}\right) \mathbf{C} \overline{\mathbf{L}}\left(x_{N}\right) \overline{\mathbf{C}}
\end{array}\right] \\
& \mathbf{L}_{(2,0)}^{*}=\left[\begin{array}{c}
\mathbf{L}\left(x_{0}\right) \mathbf{C}^{2} \overline{\mathbf{L}}\left(x_{0}\right) \\
\mathbf{L}\left(x_{1}\right) \mathbf{C}^{2} \overline{\mathbf{L}}\left(x_{1}\right) \\
\vdots \\
\mathbf{L}\left(x_{N}\right) \mathbf{C}^{2} \overline{\mathbf{L}}\left(x_{N}\right)
\end{array}\right], \\
& \mathbf{L}_{(2,1)}^{*}=\left[\begin{array}{c}
\mathbf{L}\left(x_{0}\right) \mathbf{C}^{2} \overline{\mathbf{L}}\left(x_{0}\right) \overline{\mathbf{C}} \\
\mathbf{L}\left(x_{1}\right) \mathbf{C}^{2} \overline{\mathbf{L}}\left(x_{1}\right) \overline{\mathbf{C}} \\
\vdots \\
\mathbf{L}\left(x_{N}\right) \mathbf{C}^{2} \overline{\mathbf{L}}\left(x_{N}\right) \overline{\mathbf{C}}
\end{array}\right], \\
& \mathbf{L}_{(2,2)}^{*}=\left[\begin{array}{c}
\mathbf{L}\left(x_{0}\right) \mathbf{C}^{2} \overline{\mathbf{L}}\left(x_{0}\right) \overline{\mathbf{C}^{2}} \\
\mathbf{L}\left(x_{1}\right) \mathbf{C}^{2} \overline{\mathbf{L}}\left(x_{1}\right) \overline{\mathbf{C}^{2}} \\
\vdots \\
\mathbf{L}\left(x_{N}\right) \mathbf{C}^{2} \overline{\mathbf{L}}\left(x_{N}\right) \overline{\mathbf{C}^{2}}
\end{array}\right]
\end{aligned}
$$$$
\mathbf{L}_{(0,0,0)}^{*}=\left[\begin{array}{c}
\mathbf{L}\left(x_{0}\right) \overline{\mathbf{L}}\left(x_{0}\right) \overline{\overline{\mathbf{L}}}\left(x_{0}\right) \\
\mathbf{L}\left(x_{1}\right) \overline{\mathbf{L}}\left(x_{1}\right) \overline{\overline{\mathbf{L}}}\left(x_{1}\right) \\
\vdots \\
\mathbf{L}\left(x_{N}\right) \overline{\mathbf{L}}\left(x_{N}\right) \overline{\overline{\mathbf{L}}}\left(x_{N}\right)
\end{array}\right]
$$$$
\mathbf{L}_{(1,0,0)}^{*}=\left[\begin{array}{c}
\mathbf{L}\left(x_{0}\right) \mathbf{C} \overline{\mathbf{L}}\left(x_{0}\right) \overline{\overline{\mathbf{L}}}\left(x_{0}\right) \\
\mathbf{L}\left(x_{1}\right) \mathbf{C} \overline{\mathbf{L}}\left(x_{1}\right) \overline{\overline{\mathbf{L}}}\left(x_{1}\right) \\
\vdots \\
\mathbf{L}\left(x_{N}\right) \mathbf{C} \overline{\mathbf{L}}\left(x_{N}\right) \overline{\overline{\mathbf{L}}}\left(x_{N}\right)
\end{array}\right],
$$$$
\mathbf{L}_{(1,1,0)}^{*}=\left[\begin{array}{c}
\mathbf{L}\left(x_{0}\right) \mathbf{C} \overline{\mathbf{L}}\left(x_{0}\right) \overline{\mathbf{C}} \overline{\overline{\mathbf{L}}}\left(x_{0}\right) \\
\mathbf{L}\left(x_{1}\right) \mathbf{C} \overline{\mathbf{L}}\left(x_{1}\right) \overline{\mathbf{C}}\left(x_{1}\right) \\
\vdots \\
\mathbf{L}\left(x_{N}\right) \mathbf{C} \overline{\mathbf{L}}\left(x_{N}\right) \overline{\mathbf{C}} \overline{\mathbf{L}}\left(x_{N}\right)
\end{array}\right] \text {, }
$$$$
\mathbf{L}_{(1,1,1)}^{*}=\left[\begin{array}{c}
\mathbf{L}\left(x_{0}\right) \mathbf{C} \overline{\mathbf{L}}\left(x_{0}\right) \overline{\mathbf{C L}}\left(x_{0}\right) \overline{\overline{\mathbf{C}}} \\
\mathbf{L}\left(x_{1}\right) \mathbf{C} \overline{\mathbf{L}}\left(x_{1}\right) \overline{\mathbf{C} \mathbf{L}}\left(x_{1}\right) \overline{\overline{\mathbf{C}}} \\
\vdots \\
\mathbf{L}\left(x_{N}\right) \mathbf{C} \overline{\mathbf{L}}\left(x_{N}\right) \overline{\mathbf{C L}}\left(x_{N}\right) \overline{\overline{\mathbf{C}}}
\end{array}\right],
$$

$$
\begin{aligned}
& \mathbf{L}_{(2,0,0)}^{*}=\left[\begin{array}{c}
\mathbf{L}\left(x_{0}\right) \mathbf{C}^{2} \overline{\mathbf{L}}\left(x_{0}\right) \overline{\overline{\mathbf{L}}}\left(x_{0}\right) \\
\mathbf{L}\left(x_{1}\right) \mathbf{C}^{2} \overline{\mathbf{L}}\left(x_{1}\right) \overline{\overline{\mathbf{L}}}\left(x_{1}\right) \\
\vdots \\
\mathbf{L}\left(x_{N}\right) \mathbf{C}^{2} \overline{\mathbf{L}}\left(x_{N}\right) \overline{\overline{\mathbf{L}}}\left(x_{N}\right)
\end{array}\right], \\
& \mathbf{L}_{(2,1,0)}^{*}=\left[\begin{array}{c}
\mathbf{L}\left(x_{0}\right) \mathbf{C}^{2} \overline{\mathbf{L}}\left(x_{0}\right) \overline{\mathbf{C}} \overline{\overline{\mathbf{L}}}\left(x_{0}\right) \\
\mathbf{L}\left(x_{1}\right) \mathbf{C}^{2} \overline{\mathbf{L}}\left(x_{1}\right) \overline{\mathbf{C} \mathbf{L}}\left(x_{1}\right) \\
\vdots \\
\mathbf{L}\left(x_{N}\right) \mathbf{C}^{2} \overline{\mathbf{L}}\left(x_{N}\right) \overline{\mathbf{C}} \overline{\mathbf{L}}\left(x_{N}\right)
\end{array}\right], \\
& \mathbf{L}_{(2,1,1)}^{*}=\left[\begin{array}{c}
\mathbf{L}\left(x_{0}\right) \mathbf{C}^{2} \overline{\mathbf{L}}\left(x_{0}\right) \overline{\mathbf{C}} \overline{\mathbf{\mathbf { L }}}\left(x_{0}\right) \overline{\overline{\mathbf{C}}} \\
\mathbf{L}\left(x_{1}\right) \mathbf{C}^{2} \overline{\mathbf{L}}\left(x_{1}\right) \overline{\mathbf{C}} \overline{\mathbf{L}}\left(x_{1}\right) \overline{\overline{\mathbf{C}}} \\
\vdots \\
\mathbf{L}\left(x_{N}\right) \mathbf{C}^{2} \overline{\mathbf{L}}\left(x_{N}\right) \overline{\mathbf{C}} \overline{\overline{\mathbf{L}}}\left(x_{N}\right) \overline{\overline{\mathbf{C}}}
\end{array}\right], \\
& \mathbf{L}_{(2,2,0)}^{*}=\left[\begin{array}{c}
\mathbf{L}\left(x_{0}\right) \mathbf{C}^{2} \overline{\mathbf{L}}\left(x_{0}\right) \overline{\mathbf{C}^{2}} \overline{\overline{\mathbf{L}}}\left(x_{0}\right) \\
\mathbf{L}\left(x_{1}\right) \mathbf{C}^{2} \overline{\mathbf{L}}\left(x_{1}\right) \overline{\mathbf{C}^{2}} \overline{\overline{\mathbf{L}}}\left(x_{1}\right) \\
\vdots \\
\mathbf{L}\left(x_{N}\right) \mathbf{C}^{2} \overline{\mathbf{L}}\left(x_{N}\right) \overline{\mathbf{C}^{2}} \overline{\overline{\mathbf{L}}}\left(x_{N}\right)
\end{array}\right] \text {, } \\
& \mathbf{L}_{(2,2,1)}^{*}=\left[\begin{array}{c}
\mathbf{L}\left(x_{0}\right) \mathbf{C}^{2} \overline{\mathbf{L}}\left(x_{0}\right) \overline{\mathbf{C}^{2}} \overline{\overline{\mathbf{L}}}\left(x_{0}\right) \overline{\overline{\mathbf{C}}} \\
\mathbf{L}\left(x_{1}\right) \mathbf{C}^{2} \overline{\mathbf{L}}\left(x_{1}\right) \overline{\mathbf{C}^{2}} \overline{\overline{\mathbf{L}}}\left(x_{1}\right) \overline{\overline{\mathbf{C}}} \\
\vdots \\
\mathbf{L}\left(x_{N}\right) \mathbf{C}^{2} \overline{\mathbf{L}}\left(x_{N}\right) \overline{\mathbf{C}^{2}} \overline{\overline{\mathbf{L}}}\left(x_{N}\right) \overline{\overline{\mathbf{C}}}
\end{array}\right], \\
& \mathbf{L}_{(2,2,2)}^{*}=\left[\begin{array}{c}
\mathbf{L}\left(x_{0}\right) \mathbf{C}^{2} \overline{\mathbf{L}}\left(x_{0}\right) \overline{\mathbf{C}^{2}} \overline{\overline{\mathbf{L}}}\left(x_{0}\right) \overline{\overline{\mathbf{C}^{2}}} \\
\mathbf{L}\left(x_{1}\right) \mathbf{C}^{2} \overline{\mathbf{L}}\left(x_{1}\right) \overline{\mathbf{C}^{2}} \overline{\overline{\mathbf{L}}}\left(x_{1}\right) \overline{\overline{\mathbf{C}^{2}}} \\
\vdots \\
\mathbf{L}\left(x_{N}\right) \mathbf{C}^{2} \overline{\mathbf{L}}\left(x_{N}\right) \overline{\mathbf{C}^{2}} \overline{\overline{\mathbf{L}}}\left(x_{N}\right) \overline{\overline{\mathbf{C}^{2}}}
\end{array}\right] .
\end{aligned}
$$

Then the fundamental matrix equation is gained from (5)-(13)

$$
\begin{aligned}
\sum_{k=0}^{2} \mathbf{P}_{k} \mathbf{L} \mathbf{A} & +\sum_{p=0}^{2} \sum_{q=0}^{p} \mathbf{Q}_{p q} \mathbf{L}_{(p, q)}^{*} \overline{\mathbf{A}} \\
& +\sum_{p=0}^{2} \sum_{q=0}^{p} \sum_{r=0}^{q} \mathbf{Q}_{p q r} \mathbf{L}_{(p, q, r)}^{*} \overline{\overline{\mathbf{A}}} \\
& =\mathbf{G}, \quad 0 \leq x \leq b<\infty
\end{aligned}
$$

Briefly, we can write Eq.(14) as

$$
\mathbf{W A}+\mathbf{V} \overline{\mathbf{A}}+\mathbf{z} \overline{\overline{\mathbf{A}}}=\mathbf{G},
$$

where 


$$
\begin{aligned}
& \mathbf{W}=\sum_{k=0}^{2} \mathbf{P}_{k} \mathbf{L}=\left[w_{i, j}\right] ; i, j=0,1, \ldots, N, \\
& \mathbf{V}=\sum_{p=0}^{2} \sum_{q=0}^{p} \mathbf{Q}_{p q} \mathbf{L}_{(p, q)}^{*}=\left[v_{i, j}\right]_{(N+1) \times(N+1)^{2}}, \\
& \mathbf{Z}=\sum_{p=0}^{2} \sum_{q=0}^{p} \sum_{r=0}^{q} \mathbf{Q}_{p q r} \mathbf{L}_{(p, q, r)}^{*}=\left[z_{i, j}\right]_{(N+1) \times(N+1)^{3}},
\end{aligned}
$$

Moreover, fundamental matrix equation (15) can be written in the augmented matrix form

$$
[\mathbf{W} ; \mathbf{V} ; \mathbf{Z}: \mathbf{G}] \text {. }
$$

\subsection{Matrix representation of the conditions}

Let us define the matrix form of the conditions given by (2) can be written as

$$
\begin{array}{ll}
\text { for } j=0, & \mathbf{U}_{0}=\left[y^{(0)}(0)\right]=\mathbf{L}(0), \\
\text { for } j=1, & \mathbf{U}_{1}=\left[y^{(1)}(0)\right]=\mathbf{L}(0) \mathbf{C} .
\end{array}
$$

Then, we have

$$
\begin{aligned}
\mathbf{U} & =\left[\begin{array}{l}
\mathbf{U}_{0} \\
\mathbf{U}_{1}
\end{array}\right]_{2 \times(N+1)}, \\
\mathbf{O}_{2} & =\left[\begin{array}{llll}
0 & 0 & \cdots & 0 \\
0 & 0 & \cdots & 0
\end{array}\right]_{2 \times(N+1)^{2}}, \\
\mathbf{O}_{3} & =\left[\begin{array}{llll}
0 & 0 & \cdots & 0 \\
0 & 0 & \cdots & 0
\end{array}\right]_{2 \times(N+1)^{3}},
\end{aligned}
$$

or briefly,

$$
\left[\mathbf{U} ; \mathbf{O}_{2} ; \mathbf{O}_{3}: \lambda_{j}\right]
$$

Consequently, in order to find the Laguerre coefficients $a_{n},(n=0,1, \ldots, N)$ related with the approximate solution (3) of the problem (1)-(2), by replacing the 2 row matrices (17) by the last 2 rows (or any 2 rows) of the augmented matrix (16), we obtain new augmented matrix

$$
[\widetilde{\mathbf{W}} ; \widetilde{\mathbf{V}} ; \widetilde{\mathbf{Z}}: \widetilde{\mathbf{G}}]
$$

Thence the unknown Laguerre coefficients are calculated by solving (18) 77- 8]. Therefore, the Laguerre polynomial solution can be acquired as

$$
y_{N}(x)=\sum_{n=0}^{N} a_{n} L_{n}(x) .
$$

\section{Error analysis}

Definition 1 (Residual function). We define the residual function $R_{N}\left(x_{\alpha}\right)$ for $x=x_{\alpha} \in[0, b]$

$$
\begin{aligned}
R_{N}\left(x_{\alpha}\right) & =\sum_{k=0}^{2} P_{k}\left(x_{\alpha}\right) y^{(k)}\left(x_{\alpha}\right) \\
& +\sum_{p=0}^{2} \sum_{q=0}^{p} Q_{p q}\left(x_{\alpha}\right) y^{(p)}\left(x_{\alpha}\right) y^{(q)}\left(x_{\alpha}\right) \\
& +\sum_{p=0}^{2} \sum_{q=0}^{p} \sum_{r=0}^{q} Q_{p q r}\left(x_{\alpha}\right) y^{(p)}\left(x_{\alpha}\right) y^{(q)}\left(x_{\alpha}\right) y^{(r)}\left(x_{\alpha}\right) \\
& -g\left(x_{\alpha}\right) \cong 0
\end{aligned}
$$

or

$$
R_{N}\left(x_{\alpha}\right) \leq 10^{-k_{\alpha}}, \quad \text { for } k_{\alpha} \in \mathbb{Z}^{+} .
$$

Then $\left|R_{N}\left(x_{\alpha}\right)\right|$ is called as the residual function on the interval $[0, b]$.

Theorem 1. $\left|R_{N}\left(x_{\alpha}\right)\right|$ is the residual function on the interval $[0, b]$. Then

$$
\left|\int_{0}^{b} R_{N}(x) d x\right| \leq \int_{0}^{b}\left|R_{N}(x)\right| d x
$$

So, that the upper bound of the mean error $\bar{R}_{n}$ is

$$
\left|R_{N}(x)\right| \leq \frac{\int_{0}^{b}\left|R_{N}\right|(x) d x}{b}=\bar{R}_{n} .
$$

Proof. In order to see the proof briefly, we consider the Mean Value Theorem and the definition below. Then

$$
\begin{aligned}
\left|\int_{0}^{b} R_{N}(x) d x\right| & \leq \int_{0}^{b}\left|R_{N}(x)\right| d x \\
\left|\int_{0}^{b} R_{N}(x) d x\right| & \leq b\left|R_{N}(c)\right|, \quad 0 \leq c \leq b \\
\left|\int_{0}^{b} R_{N}(x) d x\right| & \leq b\left|R_{N}(c)\right| \leq \int_{a}^{b}\left|R_{N}(x)\right| d x \\
\left|R_{N}(x)\right| & \leq \frac{\int_{0}^{b}\left|R_{N}\right|(x) d x}{b}=\bar{R}_{n}
\end{aligned}
$$




\subsection{Algorithm}

- Step 0. Input initial data: $P_{k}(x), Q_{p q}(x), Q_{p q r}(x)$ and $g(x)$. Determine the mixed conditions.

- Step 1. Set $N$ where $N \in \mathbb{N}$.

- Step 2. Construct the matrices $\mathbf{L}(x), \mathbf{C}, \overline{\mathbf{L}}(x), \overline{\mathbf{C}}, \overline{\overline{\mathbf{L}}}(x), \overline{\overline{\mathbf{C}}}$ and $\mathbf{G}$ then $\mathbf{W}, \mathbf{V}, \mathbf{Z}$.

- Step 3. Define the collocation points $x_{i}=\frac{b}{N} i, i=0,1, \ldots, N$.

- Step 4. Compute $[\mathbf{W} ; \mathbf{V} ; \mathbf{Z}: \mathbf{G}]$.

- Step 5. Compute $\left[\mathbf{U} ; \mathbf{O}_{2} ; \mathbf{O}_{3}: \lambda_{j}\right]$.

- Step 6. Construct the augmented matrix $[\tilde{\mathbf{W}} ; \tilde{\mathbf{V}} ; \tilde{\mathbf{Z}}: \tilde{\mathbf{G}}]$.

- Step 7. Input: the augmented matrix arguments, forward elimination, back substitution. Output: A (Solve the system by Gaussian elimination method).

- Step 8. Put arguments $a_{n}$ in the truncated Laguerre series form.

- Step 9. Output data: the approximate solution $y_{N}(x)$.

- Step 10. Construct $y(x)$ is the exact solution of (1).

- Step 11. Stop when $R_{N}(x) \leq 10^{-k}$ where $k \in \mathbb{Z}^{+}$. Otherwise, increase $N$ and return to Step 1.

\section{Illustrative examples}

In this section, some examples will be given to show applicability of our method. All the problems have been calculated and plotted by using Maple18 and MatlabR2014b.

Example 1. First, we consider the second-order nonlinear ordinary differential equation with quadratic terms

$y^{\prime \prime}(x)+2 y^{\prime}(x)+y(x)+y^{2}(x)-y^{\prime \prime}(x) y^{\prime}(x)=12 \exp (x)+2$

with the initial conditions

$$
y(0)=3, \quad y^{\prime}(0)=2 .
$$

The exact solution of (19)-(20) is $y(x)=1+$ $2 \exp (x)$.

Table 1. $\left|R_{N}\right|$ comparison of Example 1. for different $N$ values.

\begin{tabular}{llrc}
\hline$x$ & $\left|R_{2}\right|$ & $\left|R_{4}\right|$ & $\left|R_{5}\right|$ \\
\hline$(0.0)$ & 0.000000 & 0.000000 & 0.000000 \\
$(0.1)$ & $0.341836 E-4$ & $0.530766 E-5$ & $0.450128 E-6$ \\
$(0.2)$ & $0.280551 E-3$ & $0.281048 E-4$ & $0.194988 E-5$ \\
$(0.3)$ & $0.971761 E-3$ & $0.563571 E-4$ & $0.318105 E-5$ \\
$(0.4)$ & $0.236493 E-3$ & $0.671672 E-4$ & $0.339969 E-5$ \\
$(0.5)$ & $0.474425 E-2$ & $0.525094 E-4$ & $0.365512 E-5$ \\
$(0.6)$ & $0.842376 E-2$ & $0.476701 E-4$ & $0.530256 E-5$ \\
$(0.7)$ & $0.137505 E-2$ & $0.162679 E-3$ & $0.552450 E-5$ \\
$(0.8)$ & $0.211081 E-1$ & $0.617047 E-3$ & $0.104534 E-4$ \\
$(0.9)$ & $0.309206 E-1$ & $0.177815 E-2$ & $0.808003 E-4$ \\
$(1.0)$ & $0.436563 E-1$ & $0.420369 E-2$ & $0.282554 E-3$ \\
\hline
\end{tabular}

Example 2. Now, we consider the second-order nonlinear ordinary differential equation with cubic terms,

$y^{\prime \prime}(x)-y^{\prime}(x)\left(1-y^{2}(x)\right)+y(x)=(2+\sin (x)) \cos (x) \sin (x)+1$

with the initial conditions

$$
y(0)=y^{\prime}(0)=1 \text {. }
$$

The exact solution of (21)-(22) is $y(x)=1+$ $\sin (x)$.

Table 2. $\left|R_{N}\right|$ comparison of Example 2. for different $N$ values.

\begin{tabular}{llrc}
\hline$x$ & $\left|R_{2}\right|$ & $\left|R_{4}\right|$ & $\left|R_{6}\right|$ \\
\hline$(0.0)$ & 0.000000 & 0.000000 & 0.000000 \\
$(0.1)$ & $0.516658 E-7$ & $0.554530 E-9$ & $0.551083 E-12$ \\
$(0.2)$ & $0.213306 E-8$ & $0.243668 E-10$ & $0.241721 E-11$ \\
$(0.3)$ & $0.494797 E-8$ & $0.597486 E-10$ & $0.593143 E-11$ \\
$(0.4)$ & $0.905816 E-8$ & $0.114974 E-10$ & $0.114344 E-11$ \\
$(0.5)$ & $0.145574 E-8$ & $0.193316 E-10$ & $0.192602 E-11$ \\
$(0.6)$ & $0.215357 E-8$ & $0.298030 E-9$ & $0.297203 E-11$ \\
$(0.7)$ & $0.300782 E-7$ & $0.432339 E-9$ & $0.430863 E-10$ \\
$(0.8)$ & $0.402643 E-7$ & $0.599433 E-9$ & $0.595716 E-10$ \\
$(0.9)$ & $0.521673 E-7$ & $0.802455 E-9$ & $0.793115 E-10$ \\
$(1.0)$ & $0.658529 E-7$ & $0.444952 E-8$ & $0.234134 E-9$ \\
\hline
\end{tabular}

\section{Conclusion}

In this study, we introduce a matrix method depending on Laguerre polynomials in order to solve a class of second-order nonlinear ordinary differential equations having quadratic and cubic terms numerically. Furthermore, the error analysis is given to show the accuracy of the method. The present method and its error analysis are applied 
on some illustrative examples which have been shown by the tables.

The method has some significant advantages such as;

- The present method has short and concise computing procedure by writing the algorithm in Maple18.

- The technique gives an alternative way of solution to the second-order nonlinear ordinary differential equations which varies the other methods in literature.

- The present method has sufficient results when $\mathrm{N}$ is chosen large enough.

The method also can be developed and applied to differential functional integral equations, nonlinear functional integral equations and functional systems but some modifications are required [9][10].

\section{References}

[1] Fried, I. (1979). Numerical solution of differential equations. Academic Press, New York.

[2] Kells, L.M. (1960). Elementary differential equations. ISBN 07-033530-3.

[3] Jordan, D.W. and Smith, P. (2007). Nonlinear ordinary differential equations: an introduction for Scientists and Engineers, Fourth Edition. Oxford University Press, New York.

[4] King, A.C., Billingham, J. and Otto, S.R. (2003). Differential equations: linear, nonlinear, ordinary, partial, Cambridge University Press, New York.

[5] Rawashdeh, M.S. and Maitama, S. (2015). Solving nonlinear ordinary differential equations using the NDM. Journal of Applied Analysis and Computation, 5(1), 77-88.

[6] Yüksel, G., Gülsu, M. and Sezer, M. (2011). Chebyshev polynomial solutions of a class of second-order nonlinear ordinary differential equations. Journal of Advanced Research in Scientific Computing, 3(4), 11-24.

[7] Gürbüz, B. and Sezer, M. (2016). Laguerre polynomial solutions of a class of initial and boundary value problems arising in science and engineering fields. Acta Physica Polonica A, 130(1), 194-197.

[8] Gürbüz, B. and Sezer, M. (2014). Laguerre polynomial approach for solving Lane-Emden type functional differential equations. Applied Mathematics and Computation, 242, 255-264.

[9] Bülbül, B. and Sezer, M. (2013). Numerical solution of Duffing equation by using an improved Taylor matrix method. Journal of Applied Mathematics, 2013, 691614.

[10] Inc, M., Akgul, A. and Kılıçman, A. (2013). Numerical solutions of the second-order onedimensional telegraph equation based on reproducing kernel Hilbert space method. $A b$ stract and Applied Analysis, 2013, Hindawi.

Burcu Gürbüz received her Ph.D. from the Department of Mathematics of Celal Bayar University in 2017 under the supervision of Prof. Dr. Mehmet SEZER in Applied Mathematics. Recently, she has been awarded as "2019 Young Visiting Research Fellow" from the Embassy of France and currently, she is a post-doctoral researcher at Institution of Mathematics in Johannes Gutenberg-University Mainz. She has worked as investigator in several national and international scientific research projects. Her main research interests ordinary and partial differential equations, integral and integro differential-difference equations, numerical methods, mathematical biology models and scientific computation. Ms. Gürbüz is a member of Turkish Math Society (TMD), Society for Mathematical Biology and European Society for Mathematical and Theoretical Biology (ESMTB).

(iD http://orcid.org/0000-0002-4253-5877

Mehmet Sezer received his Ph.D. under the supervision of Prof. Dr. Süeda MORALI in the Department of Applied Mathematics at Ege University in 1982 and studied in the field of "Parabolic partial differential equations". In 1982, he was Assistant Professor at Faculty of Education (Balikesir) of Uludă̆ University, Associate Professor in 1989, in 1995 Professor at Faculty of Education of Dokuz Eylül University. In 2004, Mathematics Professor at Faculty of Science at Muğla University and since 2012, he has been Applied Mathematics Professor at Faculty of Science at Celal Bayar University. His main research interests are ordinary and partial differential equations, integral and integro differential-difference equations, delay differential equations and their numerical solutions. Prof. Sezer has been reviewer for numerous influential journals, has published research articles related to differential equations, linear algebra, analytic geometry and calculus; and has been authored over 100 papers.

(iD http://orcid.org/0000-0002-7744-2574 


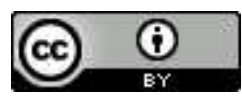

This work is licensed under a Creative Commons Attribution 4.0 International License. The authors retain ownership of the copyright for their article, but they allow anyone to download, reuse, reprint, modify, distribute, and/or copy articles in IJOCTA, so long as the original authors and source are credited. To see the complete license contents, please visit http://creativecommons.org/licenses/by/4.0/. 